\title{
Optimized Adaptive Huffmann Coding For Paper Reduction in OFDM Systems
}

\author{
Dr. Atul Suryavanshi \\ University of Engineering and Technology, Vietnam \\ atulsuryavanshi@uet.ac.vn
}

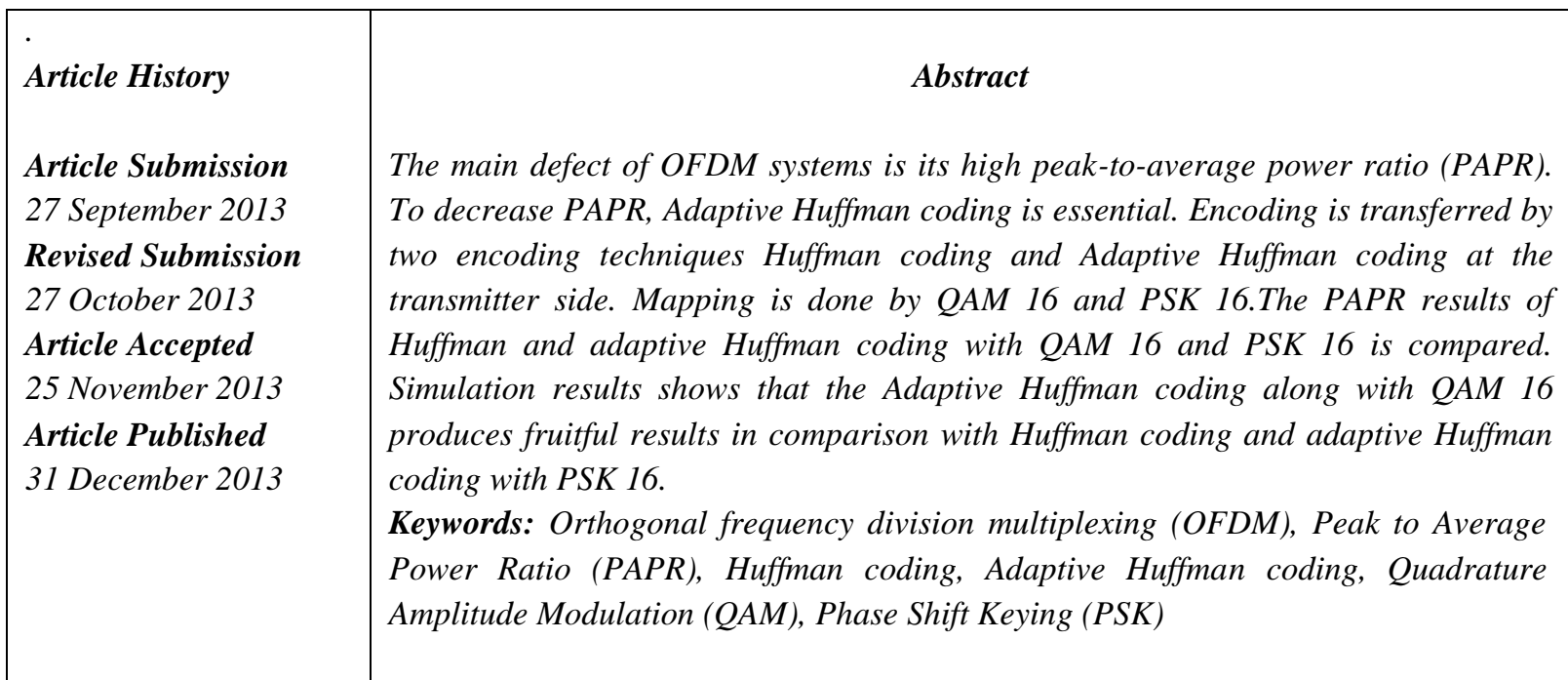

\section{Introduction}

Recent days says, acquiring high data rates are the major concern in wireless communications. So, high data rates are achieved by employing the subject of multi carrier transmission through wireless channel. There are multiple wireless communication techniques which improves data rates of the channel [1]. Among them, a specific type of multi carrier transmission method is employed which is called as OFDM. These techniques states that a frequency spectrum is classified as many sub frequency channels that are spaced equally. Also, information are broken into small packets and transmitted over a wireless frequency channel [2].

Every frequency sub band is orthogonal to the other sub band which is achieved one of the modulation techniques. The packets containing information are transmitted parallel and continuously overlapped on one another. To get high bandwidth, the carrier sub bands are superimposed and spaced adjacently (nearer to each other). The major defect of OFDM systems is its giant PAPR [3][4]. The average values are lesser than the peak values in many of the transmitted packets. There are four different effects due to high PAPR in communicated packets. First one is BER decrement, second one is effect of inter modulation on sub carrier, third one is information transferring to closely spaced frequency channels, fourth one is power amplifier faces non-linear distortion [5].

Hence, the objective is to decrease high PAPR in OFDM employed set-up. Also, there are different methods in reducing PAPR. They are tone injection, clipping, coding, partial transmit sequence are proposed in this paper. The above methods proposed are classified as namely signal scrambling methods and signal distortion methods respectively [6].

\section{Related Works}

Selected mapping(SLM), Block Coding Techniques, Interleaving Technique, Tone Reservation (TR), Tone Injection (TI), Partial Transmit Sequence(PTS) etc. In order, to decrease high peak packets in OFDM, we adopt Adaptive Huffman coding. In this encoding, decrement in the bandwidth occurs by considering codes having different lengths to encode each bit. Dynamic Huffman coding is another name for Adaptive Huffman coding established on Huffman coding which allows the symbols to develop a code to be transmitted and no 
information spread. Encoding process is done by AHC at the transmitter side. Modulation techniques used here are PSK 16, QAM 16. All the four techniques (Peak Windowing, Envelope scaling, Peak Reduction Carrier, Clipping and filtering) are compared in terms of calibrated PAPR ratio and they are observed in CCDF. AHC with QAM 16 results in having good PAPR in comparison with different techniques [7].

Large peak value in comparison with general value in the OFDM system is dependent on the existence of many unconventional modulated sub carriers [8]. Super impose of $\mathrm{N}$ complex vibrations with variant frequencies, phases and amplitudes form an envelope of OFDM information. Such high peaks effects clipping of the information at levels which are high and the impact is shown on transmitter end amplifier [9]. This operates in non- linear region creating frequency sub bands along with the initial information. Therefore, transmission is happened after the decrement of high peaks and the ratio is called Peak-to-Average Power Ratio [10].

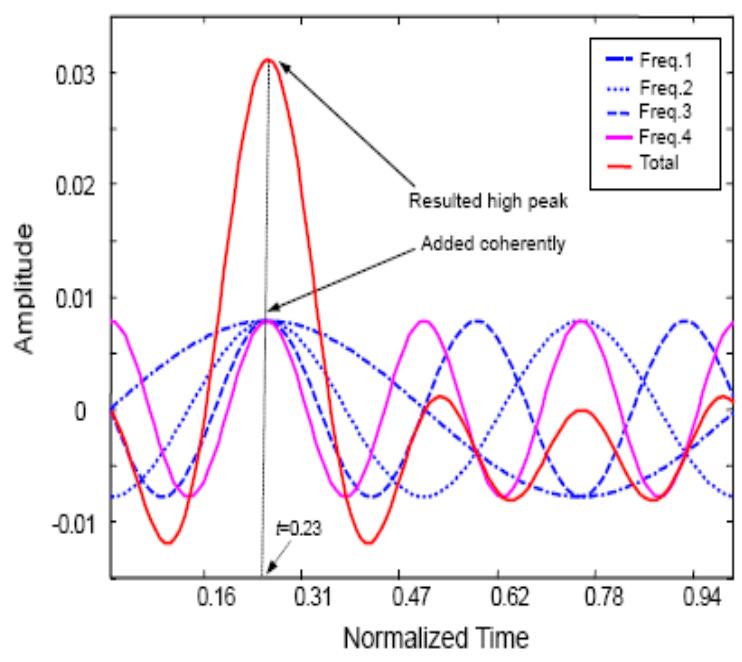

Fig.1 Schematic representation of High Peak Appearance on Multicarrier System

Mathematically PAPR can be given as

$$
P A P R=\left\{\max x(t)^{2}\right\} / E\left\{x(t)^{2}\right\}------(1)
$$

Above equation says, $\max (\mathbf{x}(t)) \mathbf{2}$ is the high peak power, $\mathbf{E}[\mathbf{x}(\mathbf{t})] \mathbf{2}$ is the mean information power.

\section{Proposed Method}

The proposed system has information bits which are serial nature at the input side are arbitrarily are converted to parallel mode. Every frequency channel is divided into different sub frequency channels in an OFDM employed system. Frequency spectrum utilization is optimized by employing frequency sub carriers. Also, inside transmitter and receiver sections we need to do more operations. This helps in converting serial to parallel information and decomposes into many different sub frequency carrier channels.

Parallel data from the S/P converter block enter as input to the Adaptive Huffman encoding block. Adaptive Huffman coding or Dynamic Huffman technique without any loss compression established on Huffman coding which allows the symbols to develop a code to be transmitted and no information spread and permits to produce the code by scanning the information at the source. Encoding of the same symbols is done differently on specific widths is a secondary method. Inside the compressed information, the understanding of the probabilities should be known to the uncompressed which is lacking in Huffman coding. Encoding the information is not only adding the bits needed. There are two methods in compressing the information; first one is used to identify the single bit frequency and establish Huffman tree, second one is to particularly compact the information. The proposed block is shown in figure 2 . 


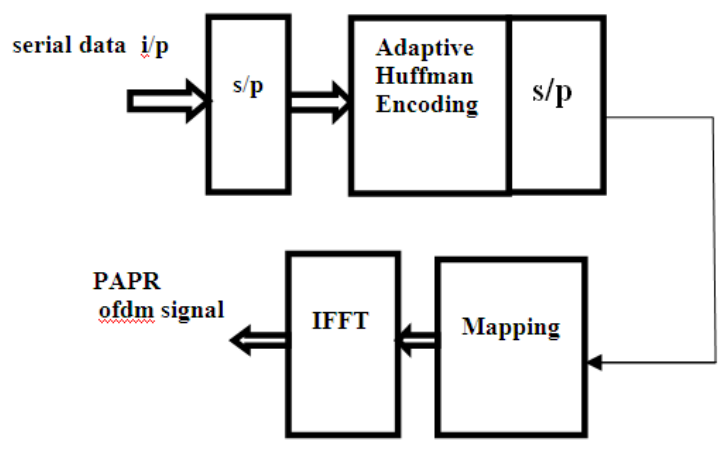

Fig. 2 Schematic representation of the Proposed Technique

Huffman coding is less productive in comparison with Adaptive Huffman coding since the tree is gradually developing. For instance, if an information starts in series which are occasional. In the tree the symbol will come down, hence encoding many bits in static Huffman coding. Whereas in AHC, the symbol is placed at the top branch in decoding process, thereby dominating high frequency information to send down the low information. Encoding the bit sequences is done by Vitter algorithm.

QAM is a modulation technique having a signal consists of two carriers orthogonal in phase (90 degrees) are modulated. Amplitude and phase variations are identified after modulation in QAM. So, it is also represented as a combination of amplitude and phase modulation. PSK is a modulation technique which is extensively used in many wireless communication systems. It helped in evolving wireless data communications and also in the increase of data rates. There are different variants of PSK in use which are available in recent years. Every variant has different drawbacks and benefits. We have to choose an optimum variant which is effective for designing radio communication systems. The algorithm is shown below

STEP 1: Every code is a tree formation which has a distinctive weight and number.

STEP 2: Bits move down, then right to left.

STEP 3: Weights are arranged according to the decrement order.

STEP 4: weight represents simply the count of bits that are transmitted.

STEP 5: Nodes having equal count of symbols are combined as a block.

STEP 6: code based on travel way from route to the node.

STEP 7: An exact technique is used for 'not yet transmitted' to transfer them.

STEP 8: The starting NYT node has the high count.

STEP 9: NYT node is transmitted, followed by code of the node.

STEP 10: Each bit in the structure of tree has a leaf node to which a code is transmitted.

STEP 11: The following process is simulated for each bit that is transmitted:

STEP 11.1: Combining child nodes along with NYT node, when the present symbol has maximum number. There are two child nodes, one is a NYT node and second one is leaf node.

STEP 11.2: Trading of the maximum count node occurs when it is not the highest.

STEP 11.3: Weight is incremented for the present node.

STEP 11.4: Move to parent node from root node and to 11.2, if root node is not identified.

\section{Simulation and Results}

After encoding, the information to spitted into many frequency channels. Each one is given for one frequency sub carrier and any basic modulation technique is used to modulate each one separately with less BER, similar bandwidth. We should not forget to do zero padding prior to modulation. To convert frequency information into time domain containing bit samples is achieved by Inverse Fourier transform. Also OFDM requires samples which are orthogonal which is obtained by IFFT. Sibling property is an important parameter in Vitter algorithm. 
The condition for sibling property is the weight of the nodes from left to right until to the top must be in descending order.i.e $\mathrm{w} 2 \leq_{\mathrm{w} 3} \leq_{\mathrm{w} 1}$, for the tree

The CCDF is given by

$$
\mathbf{P}(\mathbf{P A P R}>\mathrm{X})=1-\mathrm{P}(\mathbf{P A P R}<\mathrm{X})
$$

PAPR is calculated by using Huffman and adaptive Huffman coding. Mapping is done by QAM 16 and PSK 16 modulation. By appling two modulation schemes and two encoding techniques four combinations of outputs are produced and compared, it is shown in table 1 .

Table 1: Comparison of PAPR value

\begin{tabular}{|c|c|c|c|c|}
\hline \multirow{2}{*}{$\mathbf{N}$} & \multicolumn{2}{|c|}{ PAPR(dB) HUFFMAN } & \multicolumn{2}{c|}{ PAPR(dB) ADAPTIVE HUFFMAN } \\
\cline { 2 - 5 } & QAM16 & PSK16 & \multirow{2}{*}{ QAM16 } & PSK16 \\
\hline $\mathbf{6 4}$ & 8.1 & 8.5 & 5.8 & 6 \\
\hline $\mathbf{1 2 8}$ & 12 & 12.5 & 9 & 10 \\
\hline $\mathbf{2 5 6}$ & 15.2 & 15.5 & 12.5 & 13 \\
\hline
\end{tabular}

Simulations employing MATLAB software. OFDM with N=64 subcarriers, using QAM 16, and PSK 16 was considered Simulation results are show in figure $3 \& 4$.

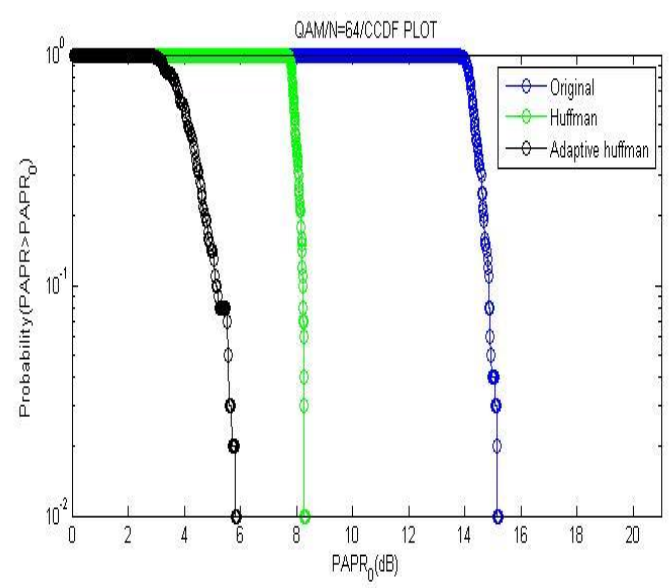

Fig.3: CCDF plot [N=64,16QAM]

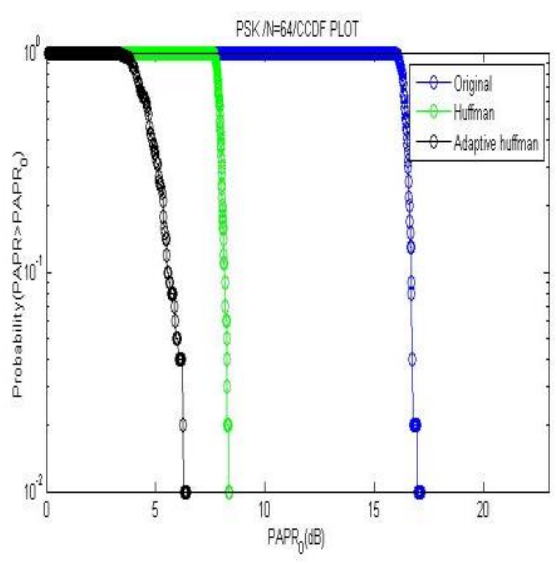

Fig.4: CCDF plot [N=64,16PSK] 
In figure $3 \&$ figure 4 the PAPR value for no coding, Huffman and adaptive Huffman was plotted. Figure 3 represents the CCDF plot for 64 subcarriers with QAM modulation for no coding the PAPR value is $15.5 \mathrm{~dB}$, for Huffman $8.1 \mathrm{~dB}$ and for adaptive Huffman the PAPR value is $5.8 \mathrm{~dB}$. In figure 3 instead of QAM, PSK modulation is used. For PSK modulation the PAPR value for Huffman and adaptive Huffman is $8.5 \mathrm{~dB}$ and $6 \mathrm{~dB}$ respectively. The figures $3 \& 4$ states that QAM modulation reduces PAPR value effectively better than the PSK modulation.

Figure 5 and figure 6 is plotted for two $\mathrm{N}$ values. In figure 5 mapping is done by QAM modulation and in figure 6 mapping is done by PSK modulation. The results shows that when the count of sub carriers $\mathrm{N}$ increases PAPR value increases.

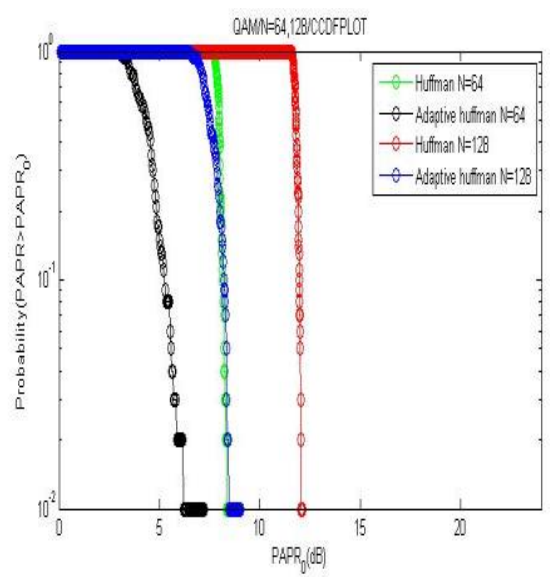

Fig 5:CCDF plot $[N=64,128, Q A M]$

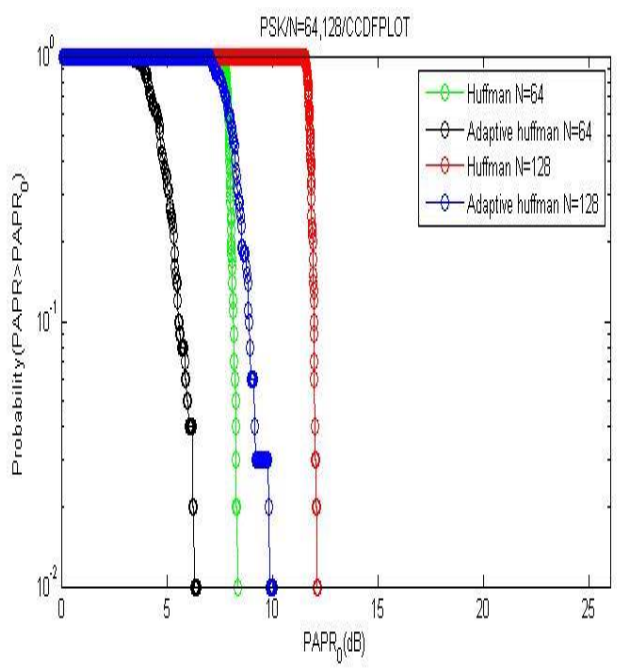

Fig.6: $C$ CDF plot $[N=64,128, P S K]$

\section{Conclusion}

The simulation result shows that Huffman coding reduces PAPR better than no coding and adaptive Huffman reduces PAPR value better than Huffman coding. QAM 16 and PSK 16 are the modulation schemes used for mapping. We have concluded that Adaptive Huffman coding reduces PAPR effectively. It is due to the advantages of adaptive Huffman coding compared to the Huffman coding, the advantages are a)only one pass over data whereas Huffman coding requires two pass over data b)code is adaptive c)no initial knowledge of source distribution. These are the main advantages of adaptive Huffman coding which supports in the reduction of PAPR value in OFDM systems. 


\section{References}

[1] Tarokh V, Jafarkhani H," On the computation and reduction of the peak-to-average power ratio in multicarrier communications", IEEE Transactions on Communications, $48 \backslash$ (1) pp. 37-44, 2000.

[2] Hyun-Bae Jheon Jong-Seon No, Fellow, IEEE,and Dond-Joon Shin, senior Member, DECEMBER 2012619 “A New PAPR Reduction Scheme Using Efficient Peak Cancellatiom for OFDM Systems, IEEE TRANSACTIONS ON BROADCASTING, VOL.58,NO. 4

[3] Dr. Pushpa R.Suri and Madhu Goel ,IJCSNS International Journal of Computer Science and Network Security, VOL.9 No.1, January 2009,"Ternary Tree \& FGK Huffman Coding Technique"

[4] Ming-Bo Lin,Member, IEEE, and Yung-Yi Chang, SEPTEMBER 20091297 “ A New Architectecture of a Two-Stage Lossless Data Compression and Decompression Algorithm" IEEE transactions on very large scale integration (VLSI) systems, VOL. 17,NO .9

[5] Hideki Ochiai,(2004)" A Novel Trellis-Shaping Design With Both Peak and Average Power Reduction for OFDM Systems" IEEE Transactions On Communications, VOL. 52, NO. 11

[6] Jia-Yu Lin, Ying Liu, and Ke-Chu Yi, MARCH 2004,"Balance of 0, 1 Bits for Huffman and Reversible Variable-Length Coding"IEEE Transactions On Communications, VOL. 52, NO. 3

[7] V. Sudha and D. Sriram Kumar, "PAPR reduction of OFDM system using PTS method with different modulation techniques," 2014 International Conference on Electronics and Communication Systems (ICECS), Coimbatore, 2014, pp. 1-5.

[8] G. S. S. Priya and B. Senthil, "An efficient scheme for PAPR reduction in Alamouti MIMO-OFDM systems," International Conference on Information Communication and Embedded Systems (ICICES2014), Chennai, 2014, pp. 1-5.

[9] C. Rajasekhar, D. Srinivasa rao, V. Yaswanth Raghava and D. Hanith, "PAPR reduction performance in OFDM systems using channel coding techniques," 2014 International Conference on Electronics and Communication Systems (ICECS), Coimbatore, 2014, pp. 1-5.

[10] J. Ferrer-Coll, B. Slimane, J. Chilo and P. Stenumgaard, "Impulsive noise detection in OFDM systems with PAPR reduction," 2014 International Symposium on Electromagnetic Compatibility, Gothenburg, 2014, pp. 523-527.

[11] Mr. Nikhil Surkar, Ms. Shriya Timande. (2012). Analysis of Analog to Digital Converter for Biomedical Applications. International Journal of New Practices in Management and Engineering, 1(03), 01 - 07. Retrieved from http://ijnpme.org/index.php/IJNPME/article/view/6

[12] Prof. Sharayu Waghmare. (2012). Vedic Multiplier Implementation for High Speed Factorial Computation. International Journal of New Practices in Management and Engineering, 1(04), 01 - 06. Retrieved from http://ijnpme.org/index.php/IJNPME/article/view/8

[13] Prof. Naveen Jain. (2013). FPGA Implementation of Hardware Architecture for H264/AV Codec Standards. International Journal of New Practices in Management and Engineering, 2(01), 01 - 07. Retrieved from http://ijnpme.org/index.php/IJNPME/article/view/11 